\title{
Photochemical Reactivity of Graphene
}

\author{
Haitao Liu, Sunmin Ryu, ${ }^{\dagger}$ Zheyuan Chen, Michael L. Steigerwald, ${ }^{*}$ Colin Nuckolls, ${ }^{*}$ and \\ Louis E. Brus* \\ Department of Chemistry and Columbia University Center for Electronics of Molecular Nanostructures, Columbia \\ University, 3000 Broadway, New York, New York 10027
}

Received May 30, 2009; E-mail: mls2064@columbia.edu; cn37@columbia.edu; leb26@columbia.edu

This paper describes a photochemical reaction between graphene and benzoyl peroxide. The reaction introduces spatially localized defects into the basal plane of graphene. Graphene, a single-layer of graphite, is an attractive material for many electrical and optoelectrical applications. ${ }^{1-3}$ Graphene is a zero gap semiconductor with extremely high charge mobility $\left(>200000 \mathrm{~cm}^{2} \mathrm{~V}^{-1} \mathrm{~s}^{-1}\right){ }^{4}$ Graphene absorbs $\sim 2 \%$ of visible light ${ }^{5}$ and has been used to make transparent flexible electronic devices ${ }^{6}$ and to replace indium tin oxide (ITO) as the transparent conducting electrodes. ${ }^{2,7}$ However, to be useful in these applications, graphene must sustain long-term photoexcitation in the presence of oxygen without degradation. Thus, understanding the photochemical reactivity of graphene is crucial in evaluating its potential as a transparent electronic material. Moreover, photochemistry may provide novel ways to covalently modify graphene, thus tailoring its chemical, electronic, and mechanical properties. ${ }^{8}$

Unlike all the small molecule aromatic compounds, graphene is a zero gap semiconductor. A recent study showed that photogenerated carriers in graphene first equilibrate among themselves through carrier-carrier scattering within $\sim 100$ fs, followed by a slower decay $(\sim 1 \mathrm{ps})$ to the Fermi level through carrier-phonon scattering. ${ }^{9}$ The lifetime of the excited carriers of graphene is $\sim 5$ orders of magnitude shorter than the fluorescence lifetimes of naphthalene and pyrene $(\sim 100 \mathrm{~ns}) .{ }^{10}$ In view of the very short lifetime, capturing the hot electrons and holes of graphene would be extremely difficult. Thus, we expect graphene to exhibit very low photochemical reactivity.

We have studied the reaction between graphene and benzoyl peroxide, a phenyl radical precursor, under laser illumination. We prepare single- and few-layer graphene microscale films by mechanical exfoliation of Kish graphite onto a silicon substrate having $300 \mathrm{~nm}$ of thermal oxide. ${ }^{1}$ Thin graphene flakes were located with an optical microscope; the exact thickness of the flake was further identified using micro Raman spectroscopy. ${ }^{11}$ In a typical reaction, a flake of single-layer graphene on the silicon substrate was immersed in a quartz cell that was filled with a toluene solution of benzoyl peroxide $(5 \mathrm{mM})$ at ambient temperature and atmosphere. The reaction was initiated by focusing an Ar-ion laser beam $(\lambda=$ $514.5 \mathrm{~nm}, 0.4 \mathrm{~mW}$ ) onto the graphene flake in the solution. A detailed description of the experiment can be found in the Supporting Information (SI).

Figure 1A shows the micro Raman spectra of the same piece of single-layer graphene before and after the photochemical treatment. After the reaction, a strong D band appeared at $1343 \mathrm{~cm}^{-1}$, which indicates that a significant number of defects were introduced into the basal plane of graphene. These defects are presumably $s p^{3}$ carbon centers produced by the phenyl radical attack on the basal plane. $^{12}$

\footnotetext{
Present address: Department of Applied Chemistry, Kyung Hee University, 1 Seocheon Kiheung, Yongin, Kyungki, 446-701, Korea.
}
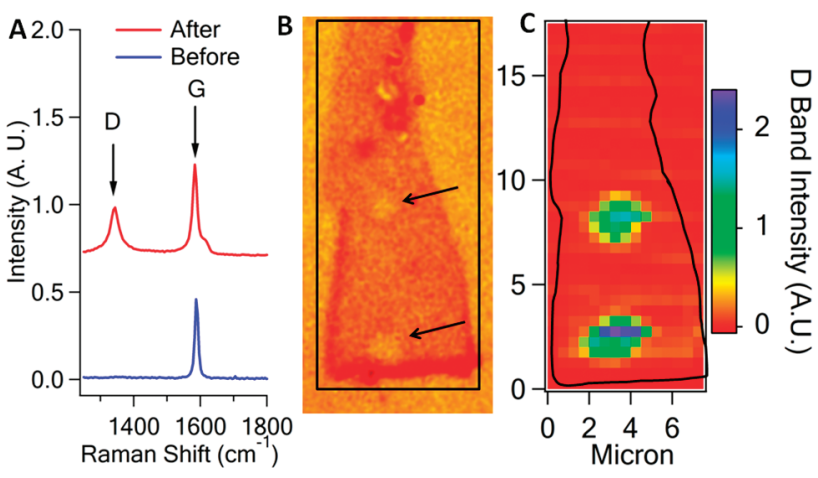

Figure 1. (A) Raman spectra $\left(\lambda_{\mathrm{ex}}=514.5 \mathrm{~nm}, 0.4 \mathrm{~mW}\right)$ of the same singlelayer graphene before and after the photochemical reaction. (B) Optical image of a single-layer graphene after the reaction. The contrast was enhanced to highlight the graphene (shown in red). The arrows indicate the holes resulted from the prolonged laser exposure. (C) Intensity map of the $\mathrm{D}$ band for the boxed area shown in (B). The black curve shows the position of the graphene flake.

Scheme 1. Reaction between Graphene and Absorbed Benzoyl Peroxide $^{a}$

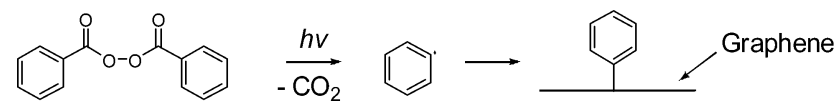

${ }^{a}$ For the sake of clarity, only one phenyl group was shown on graphene.

Scheme 1 outlines the overall reaction; no mechanistic details are implied. We find that photoexcitation is required for the reaction (see below). In addition, if the graphene flake was subject to an extended laser irradiation in benzoyl peroxide solution, micrometersized holes were observed on the basal plane with an optical (Figure 1B) and an atomic force microscope (Figure S1). We interpret the formation of the holes as the result of the photo-oxidation of the defect-rich carbon network. After the reaction, Raman imaging of the graphene flake in air showed that only the points of the laser focus give a strong D band (Figure 1C).

We have studied the effect of this photochemical reaction on the electrical properties of graphene. We carried out the photochemical reaction on a graphene field effect transistor (FET) device (Figure S2). Defects were introduced into the graphene channel between the source and drain electrodes by scanning the laser beam across the graphene flake with a computer controlled translational stage. The reaction resulted in a significant decrease (50\%) in the electrical conductivity and an increase in the hole doping level (Figure 2). The decrease in the electrical conductivity is consistent with the introduction of the $s p^{3}$ defect centers, which decreases the charge mobility. ${ }^{13} \mathrm{We}$ attribute the increase in the hole doping level to the physisorption of benzoyl peroxide on graphene. Indeed, an 

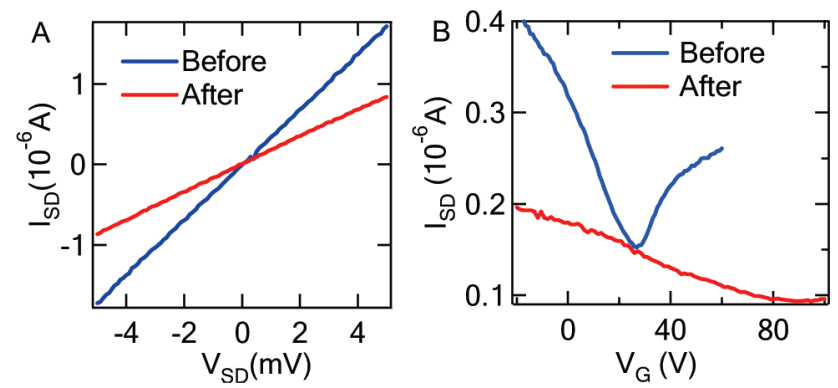

Figure 2. (A) Output and (B) transfer characteristics $\left(V_{\mathrm{SD}}=1 \mathrm{mV}\right)$ of the same graphene FET device before and after introducing defects into the graphene channel by the photochemical treatment.
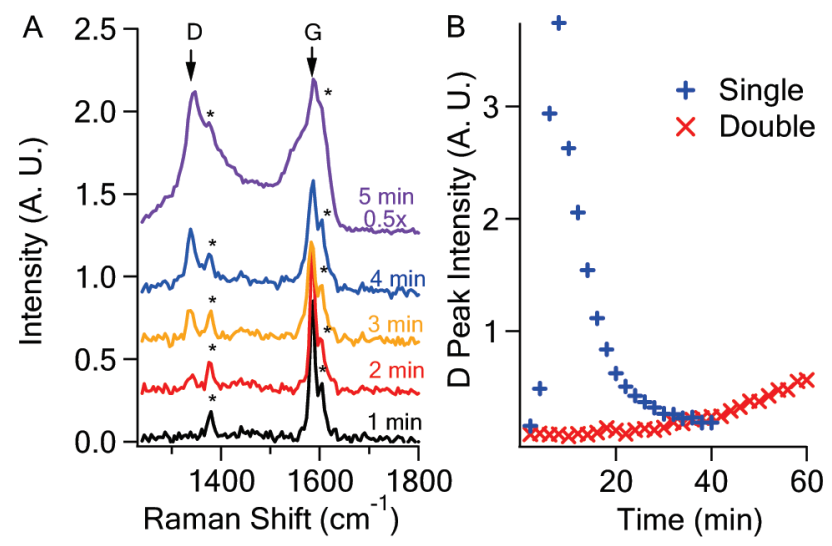

Figure 3. (A) In situ Raman spectra $\left(\lambda_{\mathrm{ex}}=514.5 \mathrm{~nm}, 0.4 \mathrm{~mW}\right)$ of a singlelayer graphene flake immersed in a solution of benzoyl peroxide in toluene $(5 \mathrm{mM})$. (*) indicates Raman peaks of toluene. (B) Time evolution of the $\mathrm{D}$ band peak intensity for a single-layer and a double-layer graphene immersed in benzoyl peroxide solution in toluene $(5 \mathrm{mM})$.

FET device treated with a benzoyl peroxide solution, but without laser exposure, showed a similar change in the hole doping level (Figure S3).

The kinetics of the photochemical reaction were studied with in situ micro Raman spectroscopy. Figure 3A shows the time evolution of the Raman spectrum of a single-layer graphene during the reaction. The reaction can be divided into two stages. In the first stage, the D band grows in intensity essentially linearly without a significant change in its width and shape (Figure 3A, 1-4 min). This stage is followed by an abrupt change in the Raman spectrum. The intensity and width of both the D and $G$ bands increased dramatically (Figure 3A, $5 \mathrm{~min}$ ). After $5 \mathrm{~min}$ of reaction, the Raman spectrum is very similar to that of graphite oxide and disordered graphitic materials, ${ }^{14}$ suggesting that the $s p^{2}$ carbon network of graphene has been broken into nanometer sized domains. ${ }^{15}$ The abrupt change in the Raman spectrum at 5 min indicates that the reaction is autocatalytic; the reactivity of graphene increases as more defect sites are formed. ${ }^{16}$ With continuing laser illumination, we observed a steady decrease in the intensities of both the D and G bands, which we attribute to the photo-oxidation of the severely damaged carbon network. The quantum yield of the reaction at the early stage was estimated to be on the order of $1.7 \times 10^{-10}$ defects per photon for a single-layer graphene (SI).

Figure 3B compares the time evolution of the D band peak intensity for a single-layer and a double-layer graphene. The reactivity of the double-layer graphene is significantly lower than its single-layer counterpart. The single-layer graphene developed a strong D band within 4 min, after which the D band intensity decreased as a result of the photo-oxidation. In contrast, we did not observe photo-oxidation of the double-layer graphene for up to $3 \mathrm{~h}$ of laser irradiation. From the growth rate of the $\mathrm{D}$ band, we estimate that the single-layer graphene is $\sim 14$ times more reactive than the double-layer graphene (SI). Our observation is consistent with recent studies on the oxidation and hydrogenation reactions of single and few-layer graphene; in both cases, single-layer graphene was found to be much more reactive than the doublelayer graphene. ${ }^{14,17,18} \mathrm{We}$ speculate that the enhanced reactivity of single-layer graphene is due to the surface induced corrugation, the lack of interlayer $\pi$ stacking, or contact of the substrate with the reacting layer. ${ }^{14,18}$ The effect of the substrate can be probed by comparing the reactivity of $\mathrm{SiO}_{2}$ supported graphene with that of mica supported and suspended ones.

We have studied the effect of oxygen, solvent, and light intensity on the reaction. We find that the defect formation is not due to oxidation by oxygen. The D band is not observed when a singlelayer graphene is irradiated with laser in air or in aerated toluene without benzoyl peroxide (Figure S4). In addition, the reaction between graphene and benzoyl peroxide still produced a strong D band under an Ar atmosphere, except that no destructive photooxidation occurs. Instead, we observed deposition of materials in the laser focus, accompanied by a very strong fluorescence background in the Raman spectrum. The deposited materials are presumably a polyphenylene type of polymer formed by the radical attack on the bound phenyl groups. ${ }^{19} \mathrm{We}$ also find that switching the solvent from toluene to benzene or $\mathrm{CCl}_{4}$ gave qualitatively the same result (Figure S5), suggesting that hydrogen abstraction of the benzylic proton of toluene does not affect the reaction to a significant degree. The effect of light intensity was studied by carrying out the reaction on two different spots of the same singlelayer graphene at laser powers of 0.4 and $0.04 \mathrm{~mW}$, respectively. The two spots are several micrometers apart to minimize any interference. We found that the D band grew at almost the same rate with respect to the number of photons (Figure S6). Since the reaction kinetics are not affected by the light intensity, we conclude that the defect formation is likely limited by a single-photon process. The lowest power level at which we were able to observe the appearance of the D band was $4 \mu \mathrm{W}\left(\sim 1600 \mathrm{~W} / \mathrm{cm}^{2}\right)$. Unfortunately, sample stage drift makes it difficult to collect kinetics data under such low light conditions.

Photoexcitation is required for the reaction. To demonstrate this, we first estimate the local temperature within the laser focus by analyzing the power dependence of the $G$ band shift, which is known to be temperature dependent. ${ }^{20} \mathrm{We}$ find that the local temperature within the laser focus is no more than $100{ }^{\circ} \mathrm{C}$ at a power level of $0.4 \mathrm{~mW}$. We then carried out thermal reactions between single-layer graphene and benzoyl peroxide in toluene at temperatures up to $110^{\circ} \mathrm{C}$. At these temperatures benzoyl peroxide readily decomposes; however, we observed no significant $\mathrm{D}$ band in these reactions (Figure S7). ${ }^{21}$

One intriguing question is how benzoyl peroxide decomposes, especially when very low laser power was used. Under such conditions, the photothermal heating is negligible; in addition, direct photolysis of benzoyl peroxide is not likely because the molecule is transparent at the laser wavelength. The only light absorbing material is graphene. We suggest that benzoyl peroxide accepts an electron from photoexcited graphene then decomposes to the phenyl radical (Figure 4). Electron transfer mediated decomposition of benzoyl peroxide is well documented. ${ }^{22}$ In particular, benzoyl peroxide can be photosensitized by small aromatic molecules, such as naphthalene. ${ }^{23}$ An exciplex formed between a photoexcited naphthalene and a ground state benzoyl peroxide is postulated to be a key player in this process. Electron transfer from the photoexcited naphthalene to benzoyl peroxide initiates its decom- 


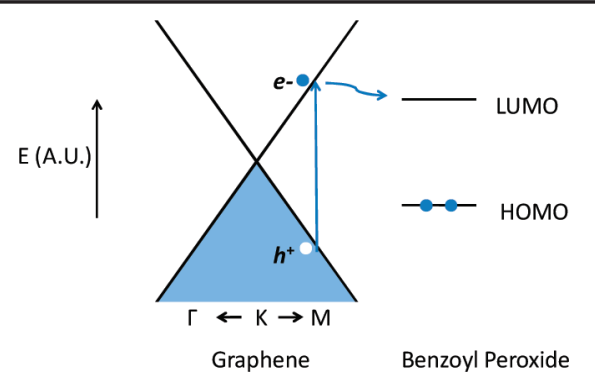

Figure 4. Hot electron transfer from photoexcited graphene to the LUMO of benzoyl peroxide. The left shows the band structure of single-layer graphene near the $\mathrm{K}$ point of the Brillouin zone. A.U., arbitrary unit.

position to benzoate and a benzoyloxyl radical. Subsequent decarboxylation of the benzoyloxyl radical produces the phenyl radical. We suggest that in our reaction graphene behaves like naphthalene. As seen above, a graphene FET device that has been immersed in a benzoyl peroxide solution showed an increased hole doping level, suggesting that benzoyl peroxide adsorbs onto the graphene surface and chemically dopes graphene by charge transfer. We suggest that the surface adsorbed benzoyl peroxide decomposes by accepting a hot electron from photoexcited graphene. The benzoyl peroxide radical anion spontaneously and irreversibly decomposes to benzoate and the benzoyloxyl radical, ${ }^{22}$ preventing the back electron transfer from the transiently reduced benzoyl peroxide to graphene. Very similar mechanisms were recently proposed for the photochemical reactions between water and graphite ${ }^{24}$ and the photooxidation of citrate on silver nanocrystals. ${ }^{25}$

To test this hot electron transfer mechanism, we have measured the reaction kinetics at three different laser wavelengths. We found that the wavelength of the laser significantly affects the reaction kinetics. At the same power level and relative to the kinetics of using a $514 \mathrm{~nm}$ laser, the reaction is $\sim 5$ times faster when using the $458 \mathrm{~nm}$ laser and is $\sim 10$ times slower when using the $633 \mathrm{~nm}$ laser (Figures S8, S9). Such a strong dependence of the reaction kinetics on the excitation energy is consistent with the hot electron transfer mechanism; an increase in the excitation energy increases the energy of the hot electron, thus increasing the rate of electron transfer to benzoyl peroxide.

Consistent with the electron transfer mechanism, we find that tert-butyl peracetate does not react with graphene under similar reaction conditions. Density functional theory calculations show that the vertical electron affinity of benzoyl peroxide is $\sim 13.2 \mathrm{kcal} /$ mol (SI). The benzoyl peroxide radical anion spontaneously decomposes to benzoate and the benzoyloxyl radical. In contrast, the radical anion of tert-butyl peracetate is unbound with respect to the neutral molecule and a free electron (SI). The difference in the vertical electron affinities of these two molecules is consistent with the reactivity difference we observed.

In conclusion, we have shown that graphene reacts with benzoyl peroxide under intense laser irradiation, producing significant defects in the basal plane. Single-layer graphene is $\sim 14$ times more reactive than double-layer graphene. After the reaction, the electrical conductivity of the graphene flake decreased while the hole-doping level increased. We suggest that a hot electron initiates an electron transfer from photoexcited graphene to the physisorbed benzoyl peroxide. This electron transfer produced a transient benzoyl peroxide radical anion, which irreversibly decomposes to produce the phenyl radical, which introduces $s p^{3}$ defect centers onto the basal plane of graphene.

Acknowledgment. We thank Prof. Nicholas J. Turro for helpful discussions. We acknowledge support from the Office of Basic
Energy Sciences, US DOE under \#DE-FG02-01ER15264 and \#FG02-98ER14861, from the NSF NIRT Award (Grant ECCS0707748), from the Nanoscale Science and Engineering Initiative of the NSF under Award Number CHE-0641523, and from the NYSTAR. H.L. was supported as part of the Center for Re-Defining Photovoltaic Efficiency Through Molecule Scale Control, US DOE, Office of Science, Office of Basic Energy Sciences under Award Number DE-SC0001085.

Supporting Information Available: Experimental details; Figure $\mathrm{S} 1$ to $\mathrm{S} 12$; compete ref $5 \mathrm{~b}$. This material is available free of charge via the Internet at http://pubs.acs.org.

\section{References}

(1) Novoselov, K. S.; Geim, A. K.; Morozov, S. V.; Jiang, D.; Zhang, Y.; Dubonos, S. V.; Grigorieva, I. V.; Firsov, A. A. Science 2004, 306, 666669.

(2) Blake, P.; Brimicombe, P. D.; Nair, R. R.; Booth, T. J.; Jiang, D.; Schedin, F.; Ponomarenko, L. A.; Morozov, S. V.; Gleeson, H. F.; Hill, E. W.; Geim, A. K.; Novoselov, K. S. Nano Lett. 2008, 8, 1704-1708.

(3) Schedin, F.; Geim, A. K.; Morozov, S. V.; Hill, E. W.; Blake, P.; Katsnelson, M. I.; Novoselov, K. S. Nat. Mater. 2007, 6, 652-655.

(4) Bolotin, K. I.; Sikes, K. J.; Jiang, Z.; Klima, M.; Fudenberg, G.; Hone, J.; Kim, P.; Stormer, H. L. Solid State Commun. 2008, 146, 351-355.

(5) (a) Mak, K. F.; Sfeir, M. Y.; Wu, Y.; Lui, C. H.; Misewich, J. A.; Heinz, T. F. Phys. Rev. Lett. 2008, 101, 196405. (b) Hernandez, Y.; et al. Nat. Nanotechnol. 2008, 3, 563-568. (c) Nair, R. R.; Blake, P.; Grigorenko, A. N.; Novoselov, K. S.; Booth, T. J.; Stauber, T.; Peres, N. M. R.; Geim, A. K. Science 2008, 320, 1308-1308.

(6) Eda, G.; Fanchini, G.; Chhowalla, M. Nat. Nanotechnol. 2008, 3, 270274 .

(7) (a) Wang, X.; Zhi, L. J.; Mullen, K. Nano Lett. 2008, 8, 323-327. (b) Li, X. L.; Zhang, G. Y.; Bai, X. D.; Sun, X. M.; Wang, X. R.; Wang, E.; Dai, H. J. Nat. Nanotechnol. 2008, 3, 538-542.

(8) (a) Ruoff, R. Nat. Nanotechnol. 2008, 3, 10-11. (b) Wang, X.; Tabakman, S. M.; Dai, H. J. Am. Chem. Soc. 2008, 130, 8152-8153. (c) Bekyarova, E.; Itkis, M. E.; Ramesh, P.; Berger, C.; Sprinkle, M.; de Heer, W. A.; Haddon, R. C. J. Am. Chem. Soc. 2009, 131, 1336-1337. (d) Lomeda, J. R.; Doyle, C. D.; Kosynkin, D. V.; Hwang, W. F.; Tour, J. M. J. Am. Chem. Soc. 2008, 130, 16201-16206. (e) Stankovich, S.; Dikin, D. A.; Dommett, G. H. B.; Kohlhaas, K. M.; Zimney, E. J.; Stach, E. A.; Piner, R. D.; Nguyen, S. T.; Ruoff, R. S. Nature 2006, 442, 282-286.

(9) Dawlaty, J. M.; Shivaraman, S.; Chandrashekhar, M.; Rana, F.; Spencer, M. G. Appl. Phys. Lett. 2008, 92, 042116.

(10) Eaton, D. F. Pure Appl. Chem. 1988, 60, 1107-1114.

(11) Ferrari, A. C.; Meyer, J. C.; Scardaci, V.; Casiraghi, C.; Lazzeri, M.; Mauri, F.; Piscanec, S.; Jiang, D.; Novoselov, K. S.; Roth, S.; Geim, A. K. Phys. Rev. Lett. 2006, 97, 187401.

(12) Bahr, J. L.; Yang, J. P.; Kosynkin, D. V.; Bronikowski, M. J.; Smalley, R. E.; Tour, J. M. J. Am. Chem. Soc. 2001, 123, 6536-6542.

(13) Elias, D. C.; Nair, R. R.; Mohiuddin, T. M. G.; Morozov, S. V.; Blake, P.; Halsall, M. P.; Ferrari, A. C.; Boukhvalov, D. W.; Katsnelson, M. I.; Geim, A. K.; Novoselov, K. S. Science 2009, 323, 610-613.

(14) Kudin, K. N.; Ozbas, B.; Schniepp, H. C.; Prud'homme, R. K.; Aksay, I. A.; Car, R. Nano Lett. 2008, 8, 36-41.

(15) Ferrari, A. C.; Robertson, J. Phys. Rev. B 2000, 61, 14095-14107.

(16) Similarly, we observed that the reactivity of the edge of the graphene flake is higher than that of the central region.

(17) Liu, L.; Ryu, S. M.; Tomasik, M. R.; Stolyarova, E.; Jung, N.; Hybertsen, M. S.; Steigerwald, M. L.; Brus, L. E.; Flynn, G. W. Nano Lett. 2008, 8, 1965-1970.

(18) Ryu, S.; Han, M. Y.; Maultzsch, J.; Heinz, T. F.; Kim, P.; Steigerwald, M. L.; Brus, L. E. Nano Lett. 2008, 8, 4597-4602.

(19) Kariuki, J. K.; McDermott, M. T. Langmuir 1999, 15, 6534-6540.

(20) Calizo, I.; Balandin, A. A.; Bao, W.; Miao, F.; Lau, C. N. Nano Lett. 2007, 7, 2645-2649.

(21) Phenyl radicals generated from diazonium compounds readily graft onto the basal plane of graphite. Such reactions, conducted in either water or polar organic solvents, are localized around the defect sites and step edges of graphite (see ref 19). The graphene flakes used here are almost defectfree and thus may show much reduced reactivity toward phenyl radicals.

(22) (a) Baron, R.; Darchen, A.; Hauchard, D. Electrochim. Acta 2006, 51, 13361341. (b) Perrin, C. L.; Wang, J. T.; Szwarc, M. J. Am. Chem. Soc. 2000, $122,4569-4572$.

(23) (a) Tokumaru, K.; Horie, K.; Simamura, O. Tetrahedron 1965, 21, 867870. (b) Urano, T.; Sakuragi, H.; Tokumaru, K. Chem. Lett. 1985, 735738 .

(24) (a) Bergeld, J.; Kasemo, B.; Chakarov, D. Phys. Chem. Chem. Phys. 2008, 10, 1151-1155. (b) Chakarov, D. V.; Gleeson, M. A.; Kasemo, B. J. Chem. Phys. 2001, 115, 9477-9483.

(25) Wu, X. M.; Redmond, P. L.; Liu, H. T.; Chen, Y. H.; Steigerwald, M.; Brus, L. J. Am. Chem. Soc. 2008, 130, 9500-9506.

JA9043906 